\title{
Self-standing aligned fiber scaffold fabrication by two photon photopolymerization
}

\author{
Hirofumi Hidai • Hojeong Jeon • David J. Hwang • \\ Costas P. Grigoropoulos
}

Published online: 9 January 2009

(C) The Author(s) 2009. This article is published with open access at Springerlink.com

\begin{abstract}
Development of materials and fabrication techniques lead the growth of three-dimensional cell culture matrices in biomedical engineering. In this work, we present a method for fabricating self-standing fiber scaffolds by two-photon polymerization induced by a femtosecond laser. The aligned fibers are $330 \mu \mathrm{m}$ long with a diameter of $6-9 \mu \mathrm{m}$. Depending on the pitch of the aligned fibers, various cell morphologies are distinguished via three-dimensional images. Furthermore, the morphologies of fibroblast cells (NIH-3T3) and epithelial cells (MDCK) on the fiber scaffolds are studied to show the effect of high curvature (3-4.5 $\mu \mathrm{m}$ radii) on cell morphology. NIH-3T3 cells that contain straight pattern of actin microfilament bundles are extended and partly wrap single fibers or tend to reside between fibers. On the other hand, MDCK cells that contain circular pattern of actin microfilament bundles cover the fiber peripheral surface exhibiting high aspect ratio elongation. These results indicate that cell morphology on fiber scaffolds is influenced by the pattern of actin microfilament bundles.
\end{abstract}

Keywords Cell morphology · Fiber scaffold . Laser manufacturing - Two-photon polymerization . Fibroblast cell $\cdot$ Epithelial cell

H. Hidai · H. Jeon • D. J. Hwang • C. P. Grigoropoulos $(\bowtie)$ Laser Thermal Laboratory, Department of Mechanical Engineering, University of California, Berkeley, CA 94720-1740, USA

e-mail: cgrigoro@me.berkeley.edu

H. Hidai

Department of Mechanical Sciences and Engineering,

Tokyo Institute of Technology,

Tokyo 152-8552, Japan

\section{Introduction}

Almost every cell in human body inhabits three-dimensional (3D) tissues that cannot be accurately represented by conventional two-dimensional (2D) culturing surfaces. In order to engineer virtually human tissue, artificial 3D cell culture scaffolds such as 3D fibrous matrices have been designed and applied to support cell and tissue growth (Hutmacher 2001; Lee et al. 2008). The scaffolds are typically fabricated with fiber based structures by fiber bonding (Mikos et al. 1993), electrospinning (Chen et al. 2007; Ji et al. 2006; Murugan and Ramakrishna 2007), weaving (Chen et al. 2004; Cooper et al. 2005), fuseddeposition modeling (Yeong et al. 2004) and carbon nanotube yarns (Galvan-Garcia et al. 2007). Fibrous structures tend to enhance cell adhesion, proliferation and differentiation functions (Lee et al. 2008). Just a few studies explored cell behavior on a single fiber or the influence of the dimensions of aligned multiple fibers on cell and tissue growth, even though groove width seems to play an important role in the control of cells on patterned surfaces (Curtis and Wilkinson 1997), largely due to lack of self-standing, position controllable fiber fabrication techniques.

In addition to developing 3D cell culture scaffolds, it is crucial to understand the interaction of biological cells with cell culture substrates for development of tissue engineering. Cell behavior, such as cell adhesion, proliferation and gene expression, can be directed by the substrate geometry and surface chemistry (Clark et al. 1990; Curtis and Wilkinson 1997; den Braber et al. 1995; Dunn and Heath 1976; Kaiser et al. 2006; Meyle et al. 1994; Rebollar et al. 2008; Rovensky et al. 1999; Rovensky and Samoilov 1994; Suh et al. 2004; Thomas et al. 1999, 2002). Various studies on these effects have been reported, most utilizing substrates 
bearing nano-scale (Rebollar et al. 2008) to micro-scale (Clark et al. 1990; Curtis and Wilkinson 1997; den Braber et al. 1995; Kaiser et al. 2006; Meyle et al. 1994) patterned surfaces as well as chemically defined surfaces (Suh et al. 2004; Thomas et al. 1999, 2002). In addition to the patterned flat surface, cylindrical substrates with high degree of curvature have been shown to affect morphogenetic response of cultured cells (Dunn and Heath 1976; Rovensky et al. 1999; Rovensky and Samoilov 1994).

In this study, two-photon induced polymerization by ultra-fast laser radiation has been used to generate selfstanding, position controllable fiber scaffolds to study cell-material interactions. Compared with conventional stereo lithography, the two-photon polymerization process can be confined to cure the photocurable resins (PR) only near the laser focal volume (Doraiswamy et al. 2006; Drakakis et al. 2006; Kawata et al. 2001), hence enabling fabrication of arbitrary three-dimensional structures. The main objective of this work is to develop high resolution self-standing, position controllable fiber fabrication techniques and to demonstrate cell culture on the fibers. The high aspect ratio fiber fabrication via two-photon photopolymerization induced by high repetition femtosecond laser irradiation is based on the combined mechanisms of self-focusing (Kewitsch and Yariv 1996), self-growing (Shoji et al. 2002) and accumulation (Hidai et al. 2008). Two-photon polymerization techniques typically utilize scanning of the laser beam to create structures by stitching the tiny voxels cured within the focal zone. In a striking difference from these studies, our technique enables fabrication of high aspect ratio $(\sim 180: 1)$ fibers with reasonable throughput as scanning is avoided (Hidai et al. 2008). Biological effects on cell shape are demonstrated using fibroblast cell lines (NIH-3T3) and epithelial cell lines (MDCK). We examine various 3D morphologies of cells cultured on fiber scaffolds and report their dependence on the cell type.

\section{Materials and methods}

\subsection{Photocurable resin}

The PR used for the fiber growth was a UV curable organic-inorganic hybrid polymer (ORMOCER ${ }^{\circledR}$, US-S4, Micro resist technology). ORMOCER ${ }^{\circledR}$ is non-toxic, biologically inert and optically transparent over the 400 $1,600 \mathrm{~nm}$ wavelength range (Doraiswamy et al. 2006).
Fig. 1 Illustration of the fabrication sequence for aligned fibers between glass plates. Two glass plates are fixed with spacer and inner side is precoated with PR (a). PR is filled between the glass plates and fibers are cured by femtosecond laser irradiation (b). Post baked and developed as a result fibers are fabricated (c). PR is cured completely by UV light irradiation, the sample is washed, sterilized FN coated, and put in Petri dish for cell culturing (d)

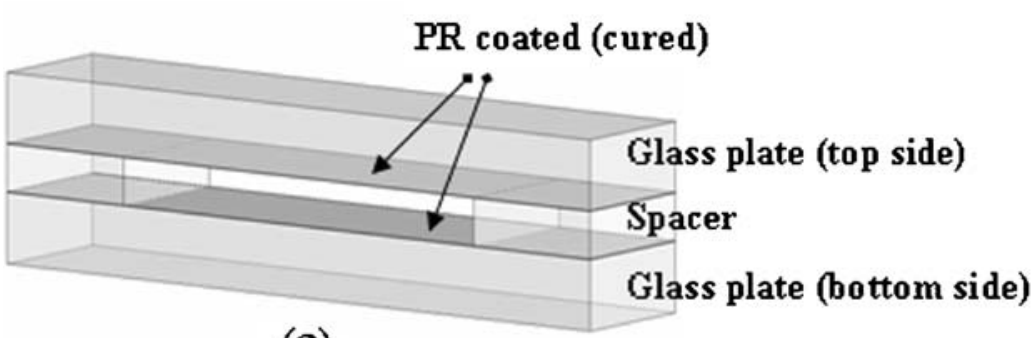

(a)

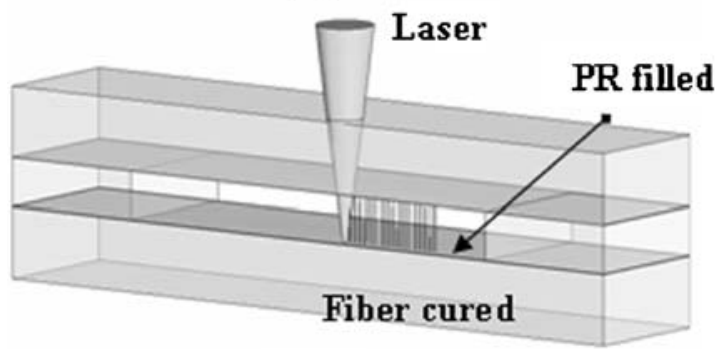

(b)

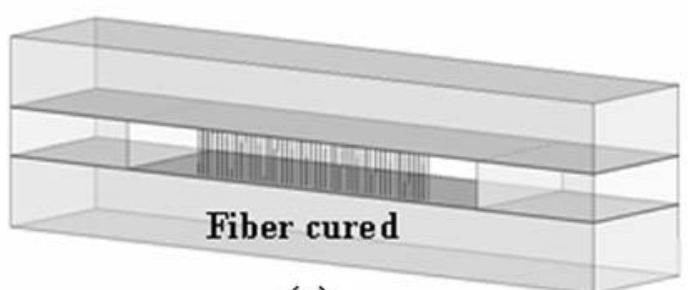

(c)

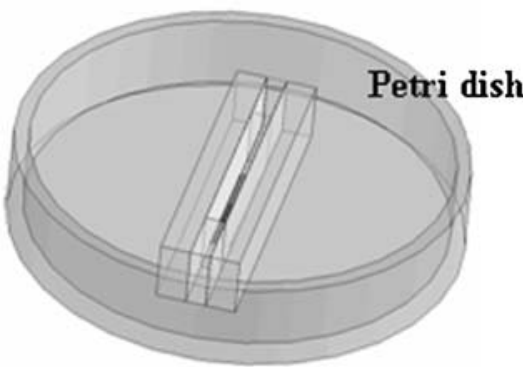

(d) 
Fig. 2 Optical and scanning electron images of fibers. (a) and (b)-(e) are optical and scanning electron images, respectively. The pitch of the fiber is $25 \mu \mathrm{m}$ and the length $0.33 \mathrm{~mm}$ (a). Images of entire fibers separated by pitch of $200 \mu \mathrm{m}$ (b). Magnified image of (b) at the top glass plate (c), in the middle (d), at the bottom glass plate (e), respectively. The scale bar indicates (a) $50 \mu \mathrm{m}$, (b) $100 \mu \mathrm{m}$ and (c)-(e) $20 \mu \mathrm{m}$
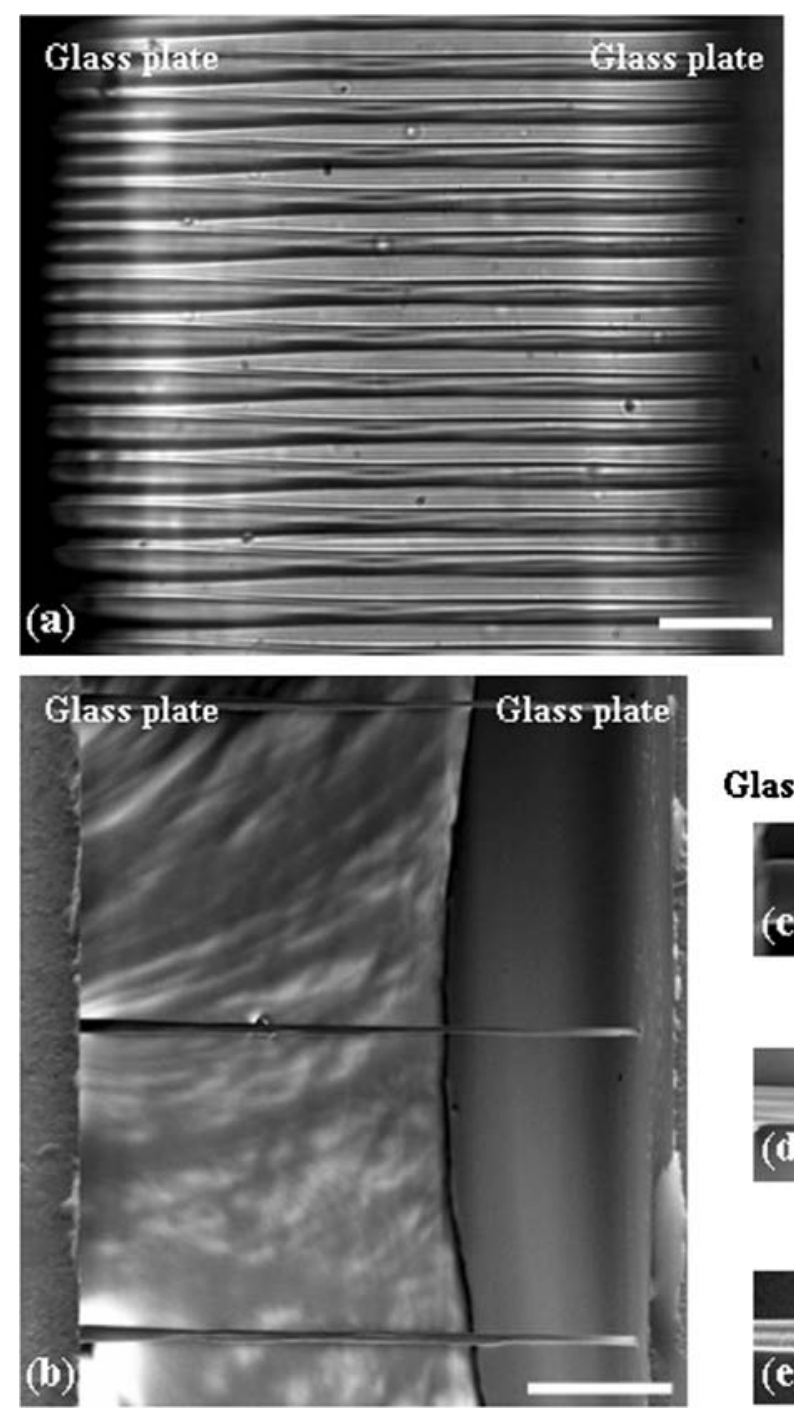

\section{Glass plate}
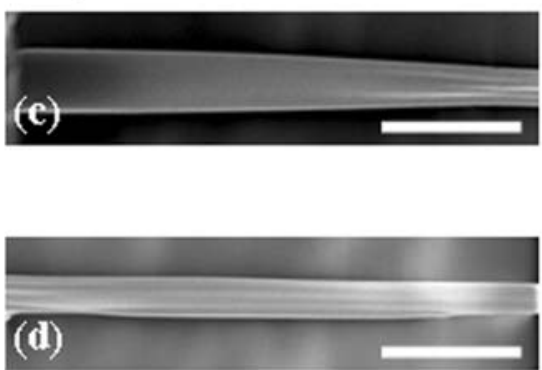

Glass plate

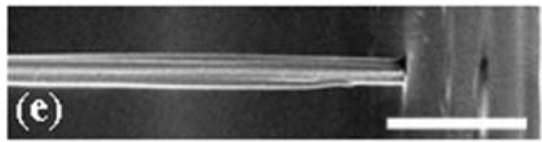

\subsection{Fabrication}

The fabrication process is illustrated in Fig. 1. Aligned fibers separated by different spacings were fabricated in the gap between two pre-PR-coated glass plates of $\sim 3 \mathrm{~mm}$ in width, $\sim 25 \mathrm{~mm}$ in length and $\sim 1 \mathrm{~mm}$ in thickness. One side of both plates was spin-coated with the PR, and then prebaked at $80^{\circ} \mathrm{C}$ for $2 \mathrm{~min}$. The two glass plates were assembled with $0.33 \mathrm{~mm}$-thick spacers (Fig. 1(a)). The PR was then cured by UV light illumination for $30 \mathrm{~min}$ and subsequently hard baked at $140^{\circ} \mathrm{C}$ for $1 \mathrm{~h}$.

After the hard bake, uncured PR was filled between the glass plates and fibers were fabricated by high-repetitionrate femtosecond laser irradiation (Fig. 1(b)). A femtosecond laser beam (pulse width: $<500 \mathrm{fs}$, repetition rate: $1 \mathrm{MHz}$, wavelength: $1,045 \mathrm{~nm}$, typical $\mathrm{M}^{2}: 1.3$, FCPA $\mu$ Jewel D-400, IMRA America, inc.) was frequencydoubled to the wavelength of $\sim 523 \mathrm{~nm}$ and focused at

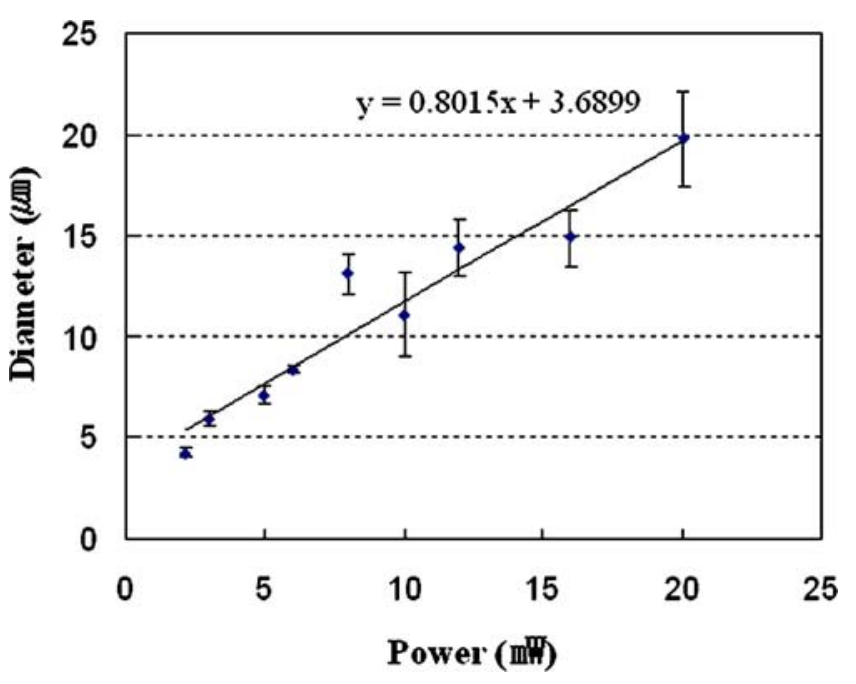

Fig. 3 The diameter of fibers is shown for different power levels of the incident laser beam. The exposure time is $200 \mathrm{~ms}$ 
$\sim 500 \mu \mathrm{m}$ below the bottom glass plate/PR interface by illuminating from the top through a $\times 5$ microscope objective (M plan apo, N.A.=0.14, Mitutoyo). The power of the laser beam emitted downstream of the objective lens was measured by a power meter and controlled by a half wave plate and a polarizing beam splitter, and was set at $\sim 3 \mathrm{~mW}$. The exposure duration was controlled by a mechanical shutter and set at $0.2 \mathrm{~s}$. The sample was placed on a motorized $\mathrm{X}-\mathrm{Y}$ stage. Fibers were fabricated at desired positions by the shutter and stages controlled by a PC.

After the laser irradiation (Fig. 1(c)), the samples were baked at $110^{\circ} \mathrm{C}$ for $10 \mathrm{~min}$, then developed with ORMO$\mathrm{DEV}^{\circledR}$ (Micro resist technology) for $30 \mathrm{~min}$, rinsed with iso-propanol (IPA) three times, deionized (DI) water with $60 \mathrm{mg} / \mathrm{mL}$ asolectin (BioChemika), dipped in DI water and exposed with UV lamp for $30 \mathrm{~min}$ to cure completely. The sample was afterward dipped in $70 \%$ ethanol to sterilize, exposed for coating to $20 \mu \mathrm{g} / \mathrm{mL}$ fibronectin (FN) (Sigma) in phosphate buffered saline (PBS) (Gibco Invitrogen) for $1 \mathrm{~h}$, and finally rinsed with PBS three times.

\subsection{Scanning electron microscopy}

Surface morphology and diameter of the fibers were characterized using a SEM (LEO 1550) at $5 \mathrm{kV}$ acceleration voltage. The sample was dried out after washing with IPA and sputter coated with gold (30 nm thick) for SEM inspection.

\subsection{Cell culture and imaging}

Cell growth and viability studies were performed using a fibroblast cell line (NIH-3T3) and epithelial cell line (MDCK). The cells were grown in Dulbecco's Modified Eagle Medium (Gibco Invitrogen), 10\% fetal bovine serum
(FBS) (Gibco Invitrogen) and $100 \mathrm{U} / \mathrm{mL}$ penicillin (Gibco Invitrogen). The cells were incubated in sterile polystyrene Petri dishes with the fiber scaffold as shown in Fig. 1(d), and stored in a $37^{\circ} \mathrm{C}$ and $5 \% \mathrm{CO}_{2}$ culture incubator.

For time-resolved image acquisition, cells were cultured on fibers and maintained at $37^{\circ} \mathrm{C}$ in $\mathrm{CO}_{2}$ independent media (Gibco Invitrogen), 10\% FBS, $100 \mathrm{U} / \mathrm{mL}$ penicillin, and 1\% GlutaMAX (Gibco Invitrogen) on a microscope stage. Images were taken every $1 \mathrm{~min}$ with a digital CCD camera (Retiga 2000R cooled, Qimaging).

After incubation for 1 day, the cells were fixed with $3.7 \%$ formaldehyde (Fisher Scientific), and then permeabilized with $0.1 \%$ TritonX-100 (Fisher Scientific). Actin cytoskeleton and nucleus were stained with $330 \mathrm{nM}$ alexa fluor 488 phalloidin (Invitrogen) for $40 \mathrm{~min}$, and $300 \mathrm{nM}$ DAPI (Invitrogen) for $4 \mathrm{~min}$, respectively. Samples were kept in PBS and turned over for upright confocal microscope observation. A 510 Meta UV/VIS confocal microscope (Zeiss) with a $\times 63$ N.A. $=1.0 \mathrm{~W}$ Plan Apochromat dipping objective lens (Zeiss) was utilized to visualize the morphology of fluorescent-stained cells on fibers. Z-scan pictures were obtained to visualize the cell morphology.

\section{Results and discussion}

\subsection{Fiber fabrication}

In-situ observation of the fiber growth showed that it commenced in the neighborhood of the geometrical focus and then self-propagated towards the light source without scanning of either the focusing lens or the sample (Hidai et al. 2008). While PR is cured only from the surface by conventional photolithography with UV laser, under femtosecond laser illumination the fiber can be cured at an

Fig. 4 Transmitted images of cells on fibers with different pitch. NIH-3T3 cells cultured for 1 day are shown on fibers with pitch of $30 \mu \mathrm{m}$ (a), (b) and $25 \mu \mathrm{m}$ (c). NIH-3T3 cells cultured on flat polymer film coated on a cover slip (d). The scale bar indicates $50 \mu \mathrm{m}$
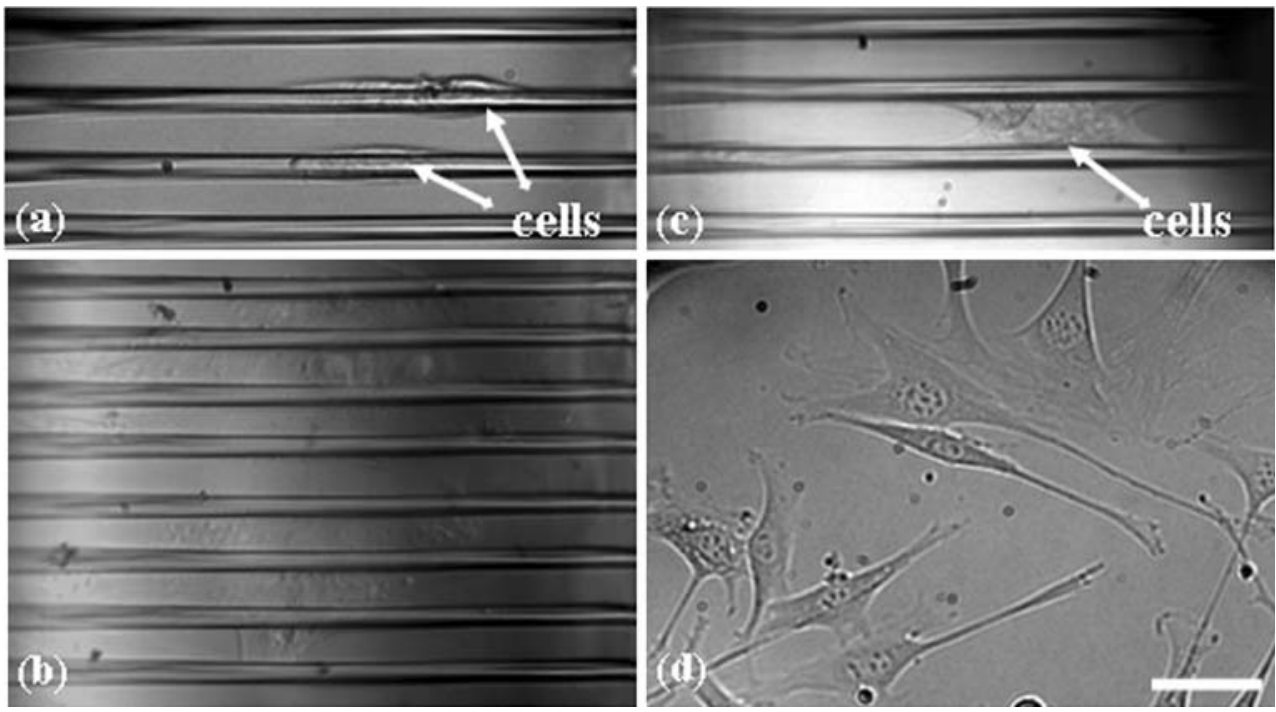
Fig. 5 Fluorescent confocal images are shown of actin cytoskeletal networks (Alexa 488phalloidin: green) and nuclei (DAPI: blue) of NIH-3T3 cells. Cells cultured for 1 day are shown on single fiber (a)-(c), wrapped around a fiber (d)-(g) and placed between fibers (h)-

(k). Three dimensional images (a), (d), (h) were obtained by image processing from layer by layer images. Confocal slices are shown at different fiber positions; on the top of the fiber (plane 1; (b), (e), (i)), the middle of the fibers (plane 2; (c), (f),

(j)) and the bottom of the fibers (plane 3; (g), (k)). NIH-3T3 cells cultured on flat polymer film coated surface on a cover slip for control (l). The scale bar indicates $20 \mu \mathrm{m}$

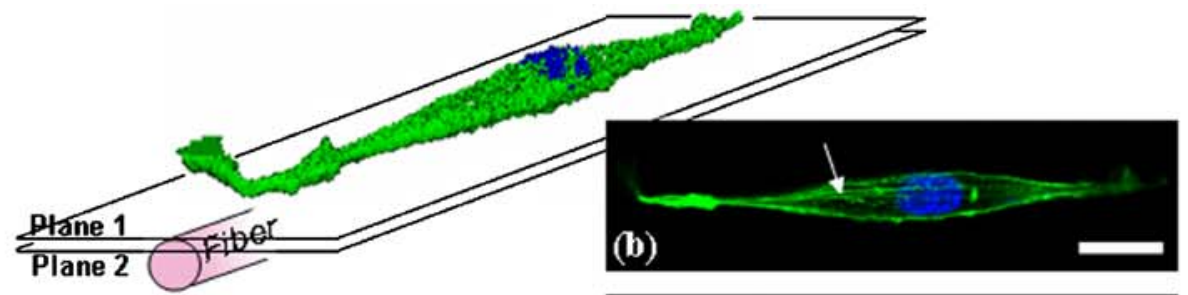

(a)
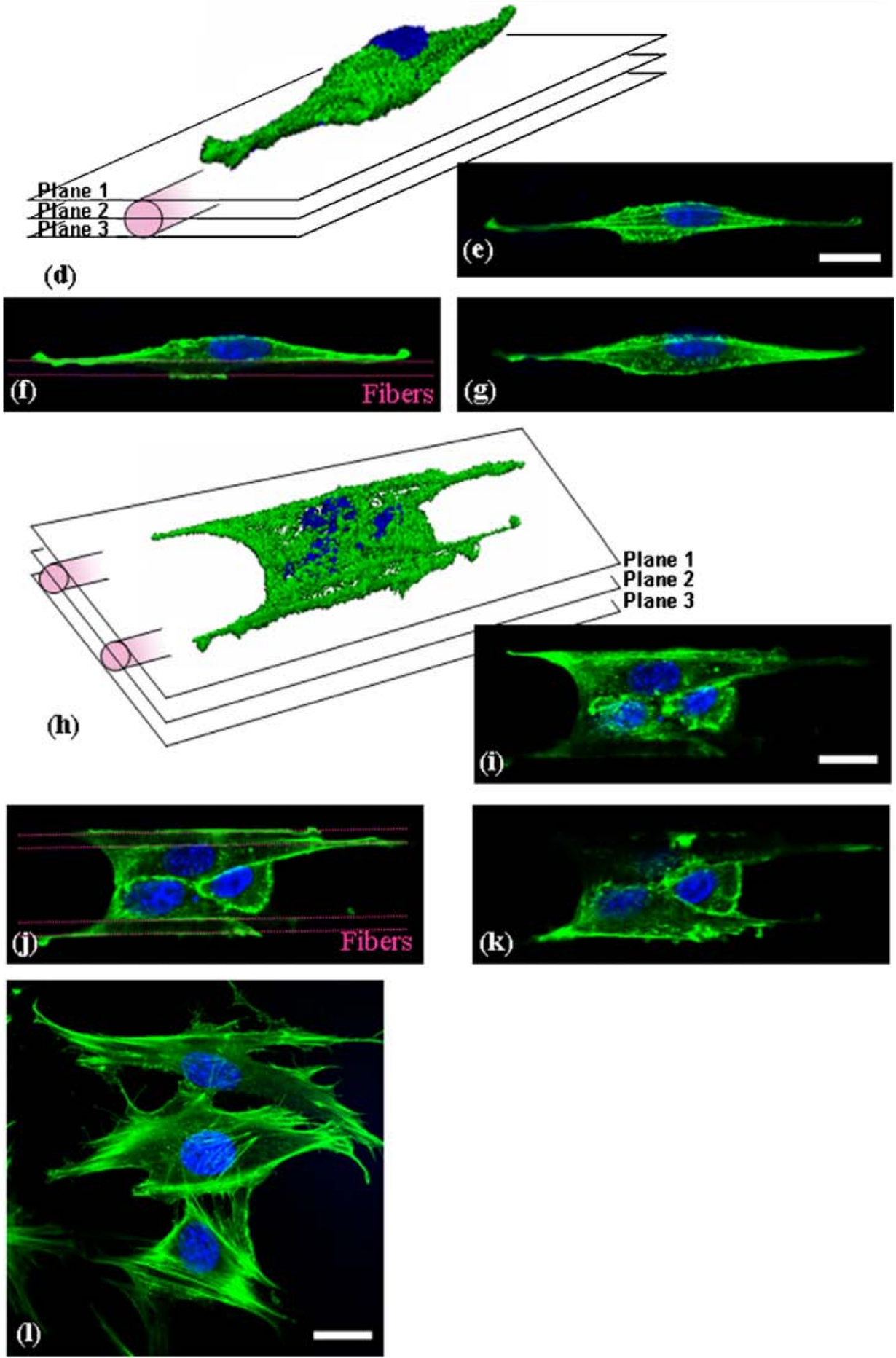

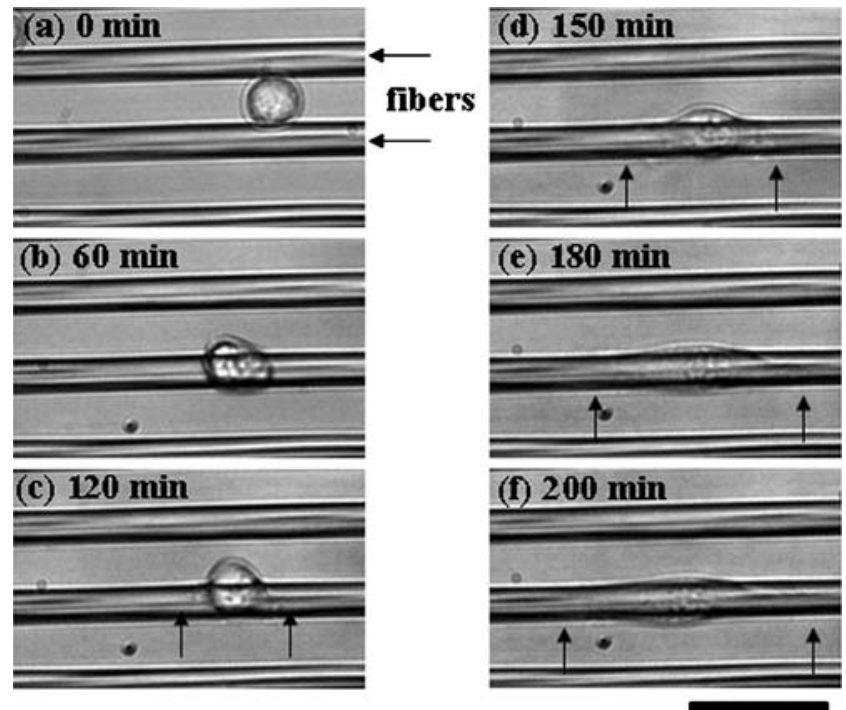

Fig. 6 Snapshots of transmitted images of the NIH-3T3 cell attachment process, after $0 \mathrm{~min}$ (a), $60 \mathrm{~min}$ (b), $120 \mathrm{~min}$ (c), $150 \mathrm{~min}$ (d), $180 \mathrm{~min}$ (e) and $200 \mathrm{~min}$ (f). Arrows indicate the cell edge. The scale bar marks $50 \mu \mathrm{m}$

arbitrary position inside the resin within the depth of focus due to two-photon absorption (Doraiswamy et al. 2006; Drakakis et al. 2006; Kawata et al. 2001).

We previously reported that fibers of $\sim 1.8 \mathrm{~mm}$ length could be readily fabricated after $\sim 1 \mathrm{~s}$ illumination with the $\sim 5 \mathrm{~mW}$ laser power when the polymer was filled in a thick glass cuvette. The fiber diameter was about $10 \mu \mathrm{m}$ in the middle and decreased toward the edges (Hidai et al. 2008). Through process optimization, fibers of $0.33 \mathrm{~mm}$ in length were fabricated bridging the two glass plates.

After laser irradiation, the sample was developed and then washed with IPA three times, once with surfactant (asolectin) solution and DI water. Figure 2(a) shows developed fibers of $\sim 0.33 \mathrm{~mm}$ length and $\sim 25 \mu \mathrm{m}$ pitch. The fibers grew upward from the surface of the bottom glass plate. The diameter at the bottom was $\sim 6 \mu \mathrm{m}$ and at the top $\sim 9 \mu \mathrm{m}$ upon illumination over a $0.2 \mathrm{~s}$ exposure time with $3 \mathrm{~mW}$ laser power. When the sample was washed with DI water instead of the surfactant solution after IPA, fibers bundled together. In order to achieve longer aligned fiber fabrication without bundling, supercritical drying technique is expected to be efficient. Details of surface morphology of the fabricated fibers by SEM are shown in Fig. 2(b)-(e). Interestingly, the grown columns were composed of smaller and directional fibers at the middle (Fig. 2(d)) and bottom (Fig. 2(e)) part, while the fiber close to the top glass plate (Fig. 2(c)) was thicker and smoother. The geometry of the fabricated fibers may depend on the nonlinear propagation characteristics of the ultra-short pulsed laser beam during the resin curing. Further studies at a fundamental level are in progress in order to unveil the detailed mechanisms. Nevertheless, the directional sub-bundle structures are expected to affect cell behavior, possibly assisting directional migration/growth along the fiber axis. The fiber diameter can be controlled by adjusting laser power and irradiation time (Hidai et al. 2008). Figure 3 shows the diameter dependence on laser power irradiated through the $\times 5$ objective for a period of $200 \mathrm{~ms}$. The calibration curve shows an almost linear relationship between fiber diameter and laser power. However, the standard deviation increases as the power rises because nonlinear propagation effects become more pronounced at high power. Fibers separated by eight different gaps, ranging from 10 to $50 \mu \mathrm{m}$ at $5 \mu \mathrm{m}$ increments were fabricated to study cell morphology.

\subsection{Cell morphology on aligned fiber scaffolds}

In order to demonstrate the biocompatibility of the fabricated fiber, fibroblast cell line (NIH-3T3) and epithelial cell line (MDCK) were cultured on the fibers coated with FN. The samples were dipped in culture solution, and then cells spread and attached on fibers.

NIH-3T3 cells after 1 day of cultivation were shown in Fig. 4. Figures 4(a)-(c) depict transmission micrographs of various cell configurations, i.e. cells located on single fibers (Fig. 4(a)), between fibers (Fig. 4(b)), cells attached to each other and situated on multiple fibers (Fig. 4(c)). As a control experiment, the PR was spin coated on a cover slip and cured by UV lamp illumination. NIH-3T3 cells also seeded on the spin coated film show a typical flat morphology (Fig. 4(d)).

Figures 5 and 7 show the typical morphology of actin cytoskeleton (green) and nuclei (blue) dyed NIH-3T3 cells and MDCK cells cultured over 1 day, respectively. Threedimensional images (Figs. 5(a), (d), (h) and 7(a), (e)) were obtained by a confocal microscope. Confocal slices are shown at different fiber positions: on the top of the fiber (plane 1; Figs. 5(b), (e), (i) and 7(b), (f)), the middle cross sectional plane (plane 2; Figs. 5(c), (f), (j) and 7(c), (g)), and the bottom of the fibers (plane 3; Figs. $5(\mathrm{~g})$, (k) and 7(d), (h)). Three dominant morphologies were observed in the case of NIH-3T3 cells: (1) elongated on a fiber, (2) partially wrapped around a fiber and (3) attached on multiple fibers. A NIH-3T3 cell, located on a single fiber (Fig. 5(a)), followed the fiber geometry forming shapes of high length/width ratio. Two actin cytoskeleton lines (arrow in Fig. 5(b)) were on the top surface of the fiber. These lines were not observed as the slice plane shifts downward by $3 \mu \mathrm{m}$, indicating that the cell conformed to the fiber surface (Fig. 5(c)). A single NIH-3T3 cell partially wrapped and was stretched along the fiber (Fig. 5(d) $-(\mathrm{g})$ ). Although Fig. 5(g) was observed through the fiber, the actin cytoskeleton could be clearly observed since the fiber 
Fig. 7 Fluorescent confocal images are shown of actin cytoskeletal networks (Alexa 488phalloidin: green) and nuclei (DAPI: blue) of MDCK cells. Cells cultured for 1 day are shown, wrapping a fiber (a)-(d) and placed between fibers (e)(h). Three dimensional images (a, e) were obtained by image processing drawn from layer by layer images. Confocal slices are shown at different fiber positions; on the top of the fiber (plane 1; (b), (f)), the middle of the fibers (plane 2; (c), (g)) and the bottom of the fibers (plane 3; (d), (h)). MDCK cells cultured on flat polymer film coated surface on a cover slip for control (k). The scale bar indicates $20 \mu \mathrm{m}$
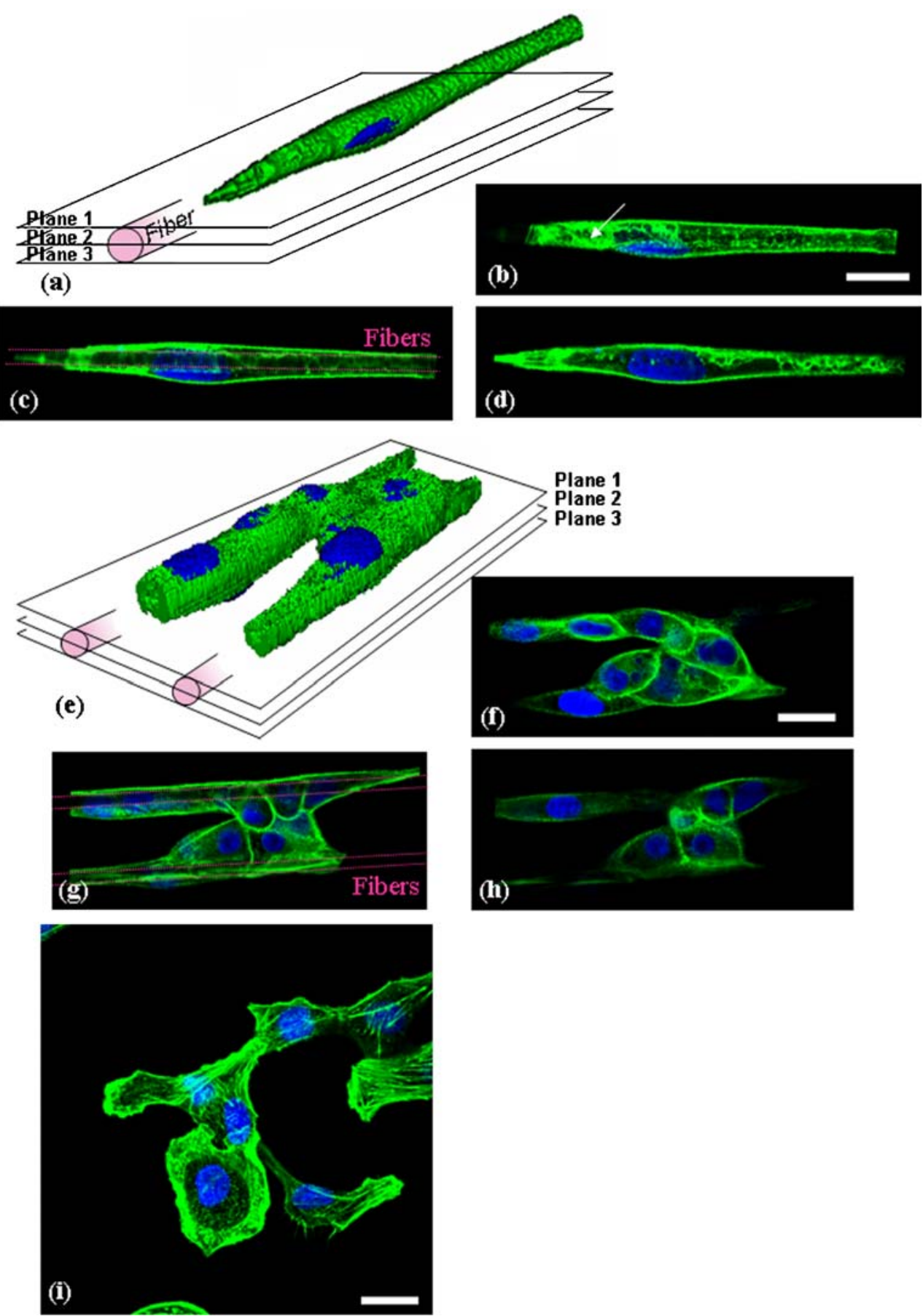

material is transparent in the visible and infrared wavelength ranges (Doraiswamy et al. 2006). Not only the cytoskeleton, but also the cell nucleus was elongated and oriented on the fiber. Dunn and Heath (1976) reported that smaller radius cylindrical substrate has more influence on elongation and orientation using glass fibers with diameters of 254 and $109 \mu \mathrm{m}$. Rovensky and Samoilov (1994) also showed that elongation and orientation of the fibroblast cells on cylindrical supports of 50 and $25 \mu \mathrm{m}$ in diameter were increased after 8 or $24 \mathrm{~h}$ of cultivation. At that cultivation stage, normal fibroblast cells presented straight actin filament bundles. Our study shows fibroblast cells on a single fiber with smaller diameter of $\sim 10 \mu \mathrm{m}$ and aligned multiple fibers. Elongation and orientation of fibroblast cells appeared to occur earlier on thinner than on thicker fibers. Time-lapsed optical transmission images shown in Fig. 6 reveal the transient extension sequence of a single cell on a single fiber. At first, a cell placed between two fibers (Fig. 6(a)) moved to attach on a single fiber (Fig. 6(b)) over which it spread and then covered 
Fig. 8 Fluorescent confocal images of actin cytoskeletal networks (Alexa 488-phalloidin: green) and nuclei (DAPI: blue) of NIH-3T3 cells located between two fibers

Fig. 9 Images of three dimensional cell morphology, cross-sectional images, and cross-sectional schematics for (a) MDCK and (b) NIH-3T3, respectively
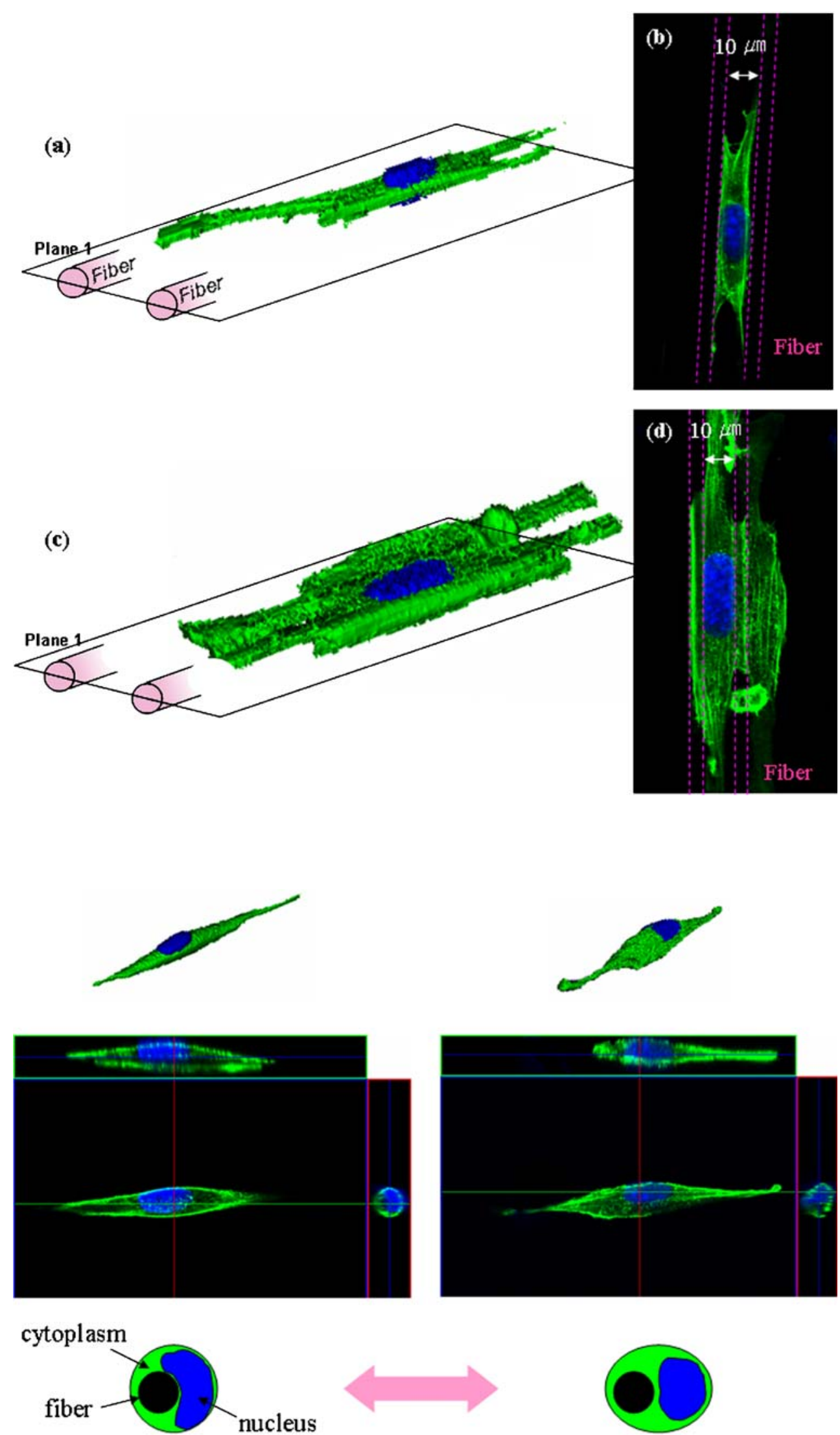

(a) MDCK
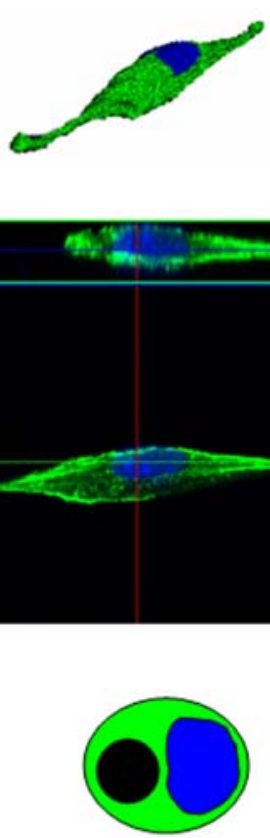

Q6.

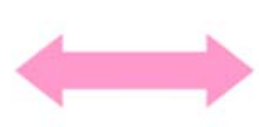

(b) NIH-3 T3 
(Fig. 6(c)-(f)). After $200 \mathrm{~min}$, the cell was elongated till about $100 \mu \mathrm{m}$. Even though straight actin fiber bundles inhibited the cell body bending, NIH-3T3 cells partially wrapped up fibers of $\sim 9 \mu \mathrm{m}$ in diameter.

On the other hand, epithelial cells exhibited different morphologies on fibers than fibroblast cells. Single MDCK cells on a fiber showed substantially strong elongation and orientation and also completely wrapped up the fiber (Fig. 7(a)-(d)). Actin cytoskeleton lines on the top surface of the fiber (arrow in Fig. 7(b)) oriented in the radial direction, and the cell body covered the fiber peripheral surface. Mikos et al. (1993) cultured hepatocytes on poly (glycolic acid) fiber of $\sim 14 \mu \mathrm{m}$ in diameter and observed that the cells covered the fiber, exhibiting similar morphologies. The difference in morphologies with respect to the cell type mirrors the pattern of actin microfilament bundles. Rovensky and Samoilov (1994) proposed that cells containing straight actin bundles resist bending and respond to the surface topography via elongation and orientation, but cells containing circular pattern of actin bundles or insufficient bundles become less resistant to bending. The difference in the pattern of actin bundles between NIH-3T3 cells and MDCK cells becomes clear when Figs. 5(1) and 7 (i) are compared. On the other hand, many MDCK cells or cell colonies also attached on multiple fibers (Fig. 7(e)-(h)) separated by smaller pitch. Cells located on fibers completely wrapped themselves around the fibers, showing similar morphology with single cells in Fig. 7(a)-(d).

It is noted that cells attain different morphologies depending on the fiber array pitch. For large array pitch (larger than the single cell diameter before extension), most cells attached on a single fiber, attaining very high length/width ratio or covering the fiber (Figs. 5(a)-(g) and 7(a)-(d)). However, when the pitch was smaller, cells or cell colonies attached on multiple fibers or connected in planar aggregates (Figs. 5(h)(k) and 7(e)-(h)). den Braber et al. (1995) cultured rat dermal fibroblasts (RDF) on silicon substrates with etched parallel surface grooves of $10 \mu \mathrm{m}$ width and spacing. They observed that the first RDF cells stretched along the grooves that were covered upon proliferation. It is noted that the ridge depth and width in that study were close to those of the PR fibers utilized in the present paper. Single NIH-3T3 cells were observed between PR fibers of 10-15 $\mu \mathrm{m}$ gap (Fig. 8). Adjacent fibers trapping cells developed contact guidance similar to groove and ridge patterned surfaces. As a result, cells trapped between fibers attained similar aligned morphologies with cells on groove/ridge patterned surface (Clark et al. 1990; den Braber et al. 1995). Single cells between fibers with gaps of over $20 \mu \mathrm{m}$ were not observed through three independent experiments. Additionally, no single MDCK cells could be located between fibers separated by $10 \mu \mathrm{m}$ gap. Because single MDCK cells could cover the fiber peripheral surface, they preferred to adhere on a single fiber than bridge across. Consequently, MDCK cells could adhere to the fiber more strongly than NIH-3T3 cells. The MDCK cell nuclei deformed to adapt to the cylindrical fiber surface, while in contrast the nuclei of NIH-3T3 cells maintained spherical shape. This is depicted in Fig. 9 that shows crosssectional images schematics for MDCK and NIH-3T3 cells.

\section{Conclusion}

We have developed a new fabrication technique to produce self-standing and controlled pitch fiber scaffolds by using two-photon polymerization effected by a high repetition rate ultra-fast laser. A cell-growing platform was chosen with the fiber diameter set at $\sim 6-9 \mu \mathrm{m}$ and the length at $\sim 0.33 \mathrm{~mm}$. Fibroblast cell lines (NIH-3T3) and epithelial cell lines (MDCK) were cultured on fibronectin coated fibers to demonstrate the biocompatibility of the fiber scaffolds and compare the cell morphologies depending on cell type and pitch of aligned fibers. This technique can be useful for fundamental level studies on the influence of microenvironment on cells and tissue engineering.

Acknowledgment Support by the U.S. National Science Foundation under grant DMI-0556363 is gratefully acknowledged.

Open Access This article is distributed under the terms of the Creative Commons Attribution Noncommercial License which permits any noncommercial use, distribution, and reproduction in any medium, provided the original author(s) and source are credited.

\section{References}

G.P. Chen, T. Sato et al., Mater. Sci. Eng. C Biomim. Supramol. Syst. 24(6-8), 861 (2004)

M. Chen, P.K. Patra et al., Tissue Eng. 13(3), 579 (2007). doi:10.1089/ ten.2006.0205

P. Clark, P. Connolly et al., Development 108(4), 635 (1990)

J.A. Cooper, H.H. Lu et al., Biomaterials 26(13), 1523 (2005). doi:10.1016/j.biomaterials.2004.05.014

A. Curtis, C. Wilkinson, Biomaterials 18(24), 1573 (1997). doi:10.1016/S0142-9612(97)00144-0

E.T. den Braber, J.E. de Ruijter et al., J. Biomed. Mater. Res. 29(4), 511 (1995). doi:10.1002/jbm.820290411

A. Doraiswamy, C. Jin et al., Acta Biomater. 2(3), 267 (2006). doi:10.1016/j.actbio.2006.01.004

T.S. Drakakis, G. Papadakis et al., Appl. Phys. Lett. 89(14), 144108 (2006). doi:10.1063/1.2359533

G.A. Dunn, J.P. Heath, Exp. Cell Res. 101(1), 1 (1976). doi:10.1016/ 0014-4827(76)90405-5

P. Galvan-Garcia, E.W. Keefer et al., J. Biomater. Sci. Polym. Ed. 18 (10), 1245 (2007). doi:10.1163/156856207782177891

H. Hidai, D.J. Hwang et al., Appl. Phys. A Mater. Sci. Process. 93(2), 443 (2008). doi:10.1007/s00339-008-4855-x

D.W. Hutmacher, J. Biomater. Sci. Polym. Ed. 12(1), 107 (2001). doi: $10.1163 / 156856201744489$

Y. Ji, K. Ghosh et al., Biomaterials 27(20), 3782 (2006). doi:10.1016/ j.biomaterials.2006.02.037 
J.P. Kaiser, A. Reinmann et al., Biomaterials 27(30), 5230 (2006). doi:10.1016/j.biomaterials.2006.06.002

S. Kawata, H.B. Sun et al., Nature 412(6848), 697 (2001). doi: $10.1038 / 35089130$

A.S. Kewitsch, A. Yariv, Opt. Lett. 21(1), 24 (1996). doi:10.1364/ OL.21.000024

J. Lee, M.J. Cuddihy et al., Tissue Eng. Part B Rev. 14(1), 61 (2008). doi:10.1089/teb.2007.0150

J. Meyle, K. Gultig et al., J. Mater. Sci. Mater. Med. 5(6-7), 463 (1994). doi:10.1007/BF00058984

A.G. Mikos, Y. Bao et al., J. Biomed. Mater. Res. 27(2), 183 (1993). doi:10.1002/jbm.820270207

R. Murugan, S. Ramakrishna, Tissue Eng. 13(8), 1845 (2007). doi:10.1089/ten.2006.0078
E. Rebollar, I. Frischauf et al., Biomaterials 29(12), 1796 (2008). doi:10.1016/j.biomaterials.2007.12.039

Y.A. Rovensky, V.I. Samoilov, J. Cell Sci. 107, 1255 (1994)

Y.A. Rovensky, L.V. Domnina et al., J. Cell Sci. 112(8), 1273 (1999)

S. Shoji, S. Kawata et al., Opt. Lett. 27(3), 185 (2002). doi:10.1364/ OL.27.000185

K.Y. Suh, J. Seong et al., Biomaterials 25(3), 557 (2004). doi:10.1016/ S0142-9612(03)00543-X

C.H. Thomas, J.B. Lhoest et al., J. Biomech. Eng. 121(1), 40 (1999). doi:10.1115/1.2798041

C.H. Thomas, J.H. Collier et al., Proc. Natl. Acad. Sci. U. S. A. 99(4), 1972 (2002). doi:10.1073/pnas.032668799

W.Y. Yeong, C.K. Chua et al., Trends Biotechnol. 22(12), 643 (2004). doi:10.1016/j.tibtech.2004.10.004 